\title{
Damage Assessment of a Structure by Using Frequency Responses Function Curvature Method and Cubic Spline Interpolation
}

\author{
Wei-Chih Su ${ }^{1, *}$ and Chiung-Shiann Huang ${ }^{2}$ \\ ${ }^{1}$ National Center for High-performance Computing, National Applied Research Laboratories, Hsinchu 30076, Taiwan \\ ${ }^{2}$ Department of Civil Engineering, National Chiao Tung University, Hsinchu 30076, Taiwan
}

\begin{abstract}
This study explores the possibility of using frequency responses function curvature method and cubic spline interpolation to locate the storeys of a building that is damaged in an earthquake, and corresponding frequency responses function curvature of storey often change in the earthquake. The frequency responses function of a building in each floor is established from the full structural dynamic responses. Next, the frequency responses function of storeys that be obtained by cubic spline interpolation would be used to detect the damage location of a building. The effectiveness of the proposed procedure is verified using numerically simulated earthquake responses of the finite element model of an eight-storey frame.
\end{abstract}

\section{Introduction}

The severe loading, such as a strong earthquake, and structural material is degraded would cause damage in a structure. The serviceability and safety of structures depend on the detection and location of structural damage. It is well-known that vibration-based damaged detection methods are simple and widely adopted [1-8]. Vibration-based damage detection methods are using the measured vibration responses of a structure to detect its damage location. Theoretically, if the stiffness and mass of a structure are changes, its dynamic properties, such as natural frequency, damping ratio, mode shapes or frequency responses function etc., are also changes. Pandey et al. employed curvature mode shapes to detect the damage in a beam, and Sampaio et al.[9] proposed frequency responses function curvature method for reducing the time-consuming on modal identification process. However, only the measured degree of freedoms could be obtained its curvature in this method.

This study using the frequency response functions curvature method and cubic spline interpolation for directly pointing out the damage location. The measured raw data will direct use for damage detection. The measured responses are in terms of acceleration or velocity. The acceleration responses are used herein. The proposed procedure is validated using the numerically simulated earthquake acceleration responses of an eightstorey finite element model.

\section{Methodology}

The dynamic responses of a linear structure satisfy the equation of motion

$$
M+C \&+K x=\mathbf{f}
$$

where $\mathbf{M}, \mathbf{C}$ and $\mathbf{K}$ are mass, damping and stiffness matrices, respectively; $\&$ and $\mathbf{x}$ are the acceleration, velocity, and displacement responses vectors of the system, and $\mathrm{f}$ is the input force vectors. Applying Fourier transform into Eq.(1) and denoting $\mathscr{F}\{\mathbf{x}\}=\overline{\mathbf{X}}(\omega)$

$$
\overline{\mathbf{X}}(\omega)=\mathbf{H}(\omega) \overline{\mathbf{F}}(\omega)
$$

where, $\quad \mathbf{H}(\omega)=\left(-\mathbf{M} \omega^{2}+i \mathbf{C} \omega+\mathbf{K}\right)^{-1}$ is well-known frequency response function. The frequency response function can be calculated by applying the weighted least-square approach on Eq. (2).

The frequency responses function curvature method is an extension of the curvature mode shapes method to all frequencies in the measurement range and not just considers the modal frequencies. In fact, the operation of this method is similar to the curvature mode shapes method. The curvature for each frequency is given by

$$
h^{\prime \prime}(\omega)_{i, j}=\left[h(\omega)_{i+1, j}-2 h(\omega)_{i, j}+h(\omega)_{i-1, j}\right] / l^{2}
$$

where $h_{i, j}$ is the receptacle FRF measured at the $i^{\text {th }}$ degree of freedom for a force input at location $j$. 
The absolute difference between the FRF curvatures of the damaged and undamaged structure at the $i^{\text {th }}$ degree of freedom, along the chosen frequency range, is calculated, for an applied force at point $j$, by the following equation

$$
\Delta h_{i, j}^{\prime \prime}=\sum_{\omega}\left|h_{d}^{\prime \prime}(\omega)_{i, j}-h_{u}^{\prime \prime}(\omega)_{i, j}\right|
$$

where, $h_{d}^{\prime \prime}(\omega)_{i, j}$ and $h_{u}^{\prime \prime}(\omega)_{i, j}$ are denoting as the curvature of damaged and undamaged structures, respectively. Finally, the each force location will be summed up and yielded

$$
S(i)=\sum_{j} \Delta h_{i, j}^{\prime \prime}
$$

However, the curvature of the FRF calculated by Eq. (3) is difficult to apply on boundary degree of freedoms. Besides, this procedure that used to detect the stiffness change between the $i-1^{\text {th }}$ and $i^{\text {th }}$ degree of freedoms is indirectly. To address these issues, the spline interpolation will be used to the curvature between the $i$ $1^{\text {th }}$ and $i^{\text {th }}$ degree of freedoms.

A cubic spline is a spine constructed of piecewise third-order polynomials which pass through a set of $\mathrm{m}$ given points. The frequency responses function between the $i-1^{\text {th }} \mathrm{h}$ and $i^{\text {th }}$ degree of freedoms for a force input at location $j$, denoted by $h(\omega)_{i-1 / 2, j}$, can be easy obtained by the cubic spline interpolation. Then, the FRF curvature between the $i-1^{\text {th }}$ and $i^{\text {th }}$ degree of freedoms for a force input at location $j$ is given by

$$
\begin{gathered}
h^{\prime \prime}(\omega)_{i-1 / 2, j}=4\left[h(\omega)_{i, j}-2 h(\omega)_{i-1 / 2, j}+h(\omega)_{i-1, j}\right] / l^{2} \\
\text { D.I.(i) }=S(i-1 / 2) / S\left(i_{\max }-1 / 2\right) \quad i=1,2, \mathrm{~L}, I
\end{gathered}
$$

$\Delta h_{i-1 / 2, j}^{\prime \prime}$ and $S(i-1 / 2)$ can be calculated by the same procedure. Finally, the damage index can be defined as

$$
\text { D.I. }(i)=S(i-1 / 2) / S\left(i_{\max }-1 / 2\right) \quad i=1,2, \mathrm{~L}, I
$$

Here $i_{\max }$ is the maximum order location, and $I$ is the measured degree of freedoms.

\section{Numerical Verification}

To demonstrate the feasibility of the proposed procedure, the finite element model of eight-storey frame was built and this frame was subjected to a simulated 1999 Chi-chi earthquake at its base in $\mathrm{X}$ direction (see Fig. 1). The eight-storey frame under consideration was $1.5 \mathrm{~m}$ long, $1.1 \mathrm{~m}$ wide and $1.06 \mathrm{~m}$ high. The simulation was conducted at $5 \%$ of the modal damping ratio. Plates were fixed on each floor, such that the total mass of frame at each floor was approximately $6.732 \mathrm{~kg}$. The columns have rectangular sections of $2.5 \times 15$, with units in $\mathrm{cm}$. This original frame was defined as "frame A". And "frame B" was created by replacing each column on the first storey with columns of rectangular sections of 2.5x13.5. Each frame was subjected to base excitations of the 1999 Chi-Chi earthquake. The acceleration responses of the base and all floors at $\mathrm{t}=5 \sim 45$ seconds were used in evaluating frequency responses function for the frames. Fig. 1 displays the time history of base excitation and acceleration responses in $\mathrm{X}$ direction of "frame A" subject the Chi-chi earthquake. Fig.1 also shows the FRFs of the "frame A" and "frame B", plotted for a frequency range of $0-20 \mathrm{~Hz}$, measured on each floor for an input force on the base.
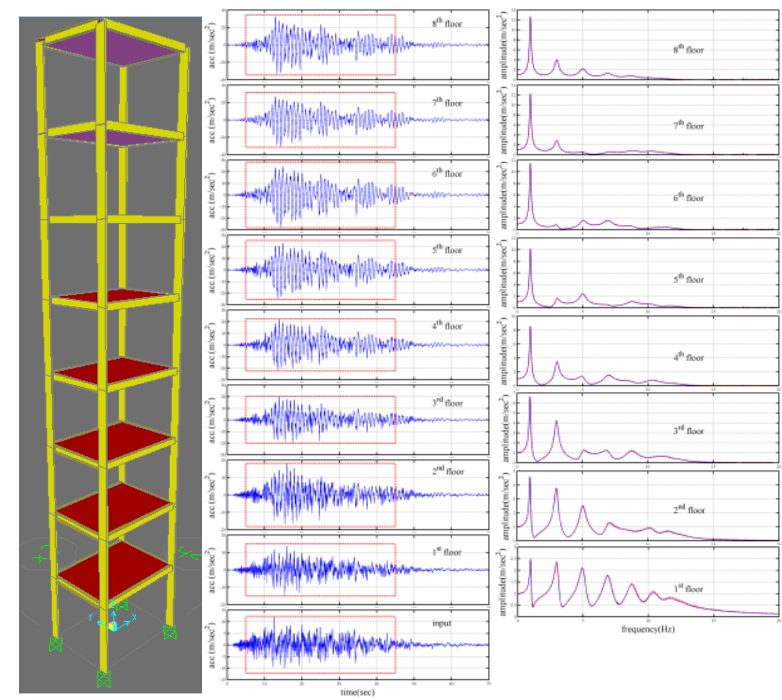

Fig. 1. Schematic diagram of an eight-storey frame and its simulation responses

This paragraph further applied this procedure to the calculated frequency responses function. Fig. 2 compares the results obtained using the presented method and the traditional frequency response function curvature method. The results in Fig. 2 are normalized the variation of the curvature of the FRFs between the "frame A" and "frame B". In theoretically, the normalized indexes induced by the "FRFCM only" in the observation points nearby the damaged elements are close to one. Fig. 2 clearly demonstrates the ability of the both method in identifying the storey in the reference structure. However, it is directly to locate the storey with stiffness reduce in the proposed procedure.

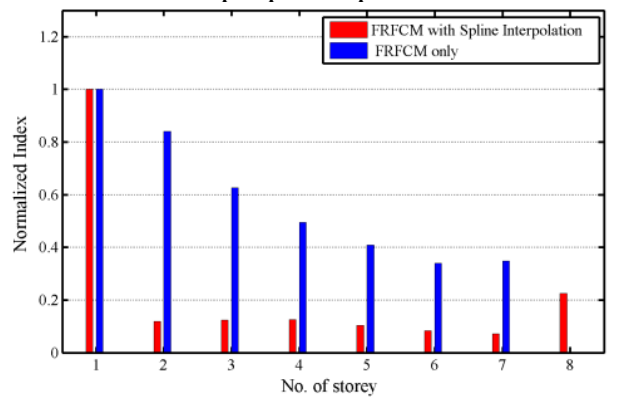

Fig. 2. Normalized index. 
The success of the proposed procedure, as revealed in capturing the difference in properties of numerical models, demonstrates the practical applicability of this procedure to a shear building model.

\section{Conclusions}

This study developed a simple and efficient approach for locating damaged storeys in a structure, based on the fact that damage to a storey changes the frequency responses function curvature of the structure. The frequency response functions are directly estimated from the measured acceleration responses. Next, the FRFs curvature between the observed locations will be obtained by applying the cubic spline interpolation on the FRFs. Comparing the FRFs curvature in the current state with those in the undamaged state enables damaged storeys to be accurately located.

The proposed approach was demonstrated on an eight-storey finite element model under earthquake excitation. A finite element model with single-site damage was considered. The proposed approach was validated by successfully identifying damaged storeys by processing numerically simulated responses. The proposed procedure did provide more directly locating the damaged storeys than the process without the cubic spline interpolation.

\section{Acknowledgement}

The authors would like to thank the Ministry of Science and Technology of the Republic of China, Taiwan, for financially supporting this research under Contract No. MOST 106-2622-M-492-001-CC2.

\section{References}

1. H.G., Natke, Probab Eng Mech. 3(1), 28-35 (1988).

2. M. Imregun, W.J. Visser, Shock Vib Dig. 23, 9-20 (1999).

3. J.E. Mottershead, M.I. Friswell, J Sound Vib. 167(2), 347-75 (1993).

4. O.S. Salawu, Eng Struct. 19(9), 718-2 (1997).

5. S.W. Doebling, C.R. Farrar, Shock Vib Dig. 30(2), 91-105 (1998).

6. C.r., Farrar, S.W. Doebling, D.A. Nix, Philosophical Transactions of the Royal Society. Math Phys Eng Sci. 359(1778), 131-50 (2001).

7. P.C. Chang, A. Flatau, S.C. Liu, Struc Health Monit, Int J. 2(3), 257-67 (2003).

8. J.M.W. Brownjohn, Philosophical Transactions of the Royal Society. Math Phys Eng Sci. 365(1851), 589-622 (2007).

9. R.P.C. Sampaio, N.M.M. Maia, J.M.M. Silva, J Sound Vib. 226(5), 1029-42 (1999). 